\title{
BUNCH LENGTH CONTROL IN DAФNE BY A HIGHER HARMONIC CAVITY
}

\author{
M. Migliorati, L. Palumbo, M. Zobov
}

\section{Introduction}

The longitudinal size of a single bunch in the DAФNE storage rings is of crucial importance for reaching high luminosity. To avoid geometrical luminosity reductions (the so-called "hour-glass effect"), the bunch length has to satisfy the empirical rule[1]:

$$
\sigma_{z} \leq \frac{\beta_{y}}{1.5}
$$

where $\beta_{y}$ is the vertical betatron function at the interaction point.

On the other hand, a too short bunch produces strong parasitic losses, fast mode-coupling instabilities, and short Touschek life-time. For DAФNE, a bunch length $\sigma_{Z}=3 \mathrm{~cm}$ has been chosen as a reasonable compromise.

Preliminary calculations on bunch lengthening in DAФNE have shown that the final bunch length is less than $3 \mathrm{~cm}$, and additional lengthening may be necessary[2]. This can be done, for example, by decreasing the main RF voltage. However, this implies a substantial reduction in the acceptance, which leads to a reduction in Touschek life-time. Therefore an alternative method to control the bunch length should be proposed.

The lack of a reliable theory describing the strong turbulent lengthening regime (expected for DAФNE) is another reason to develop a system able to cope with any unforeseen effect in beam behavior which may shorten or lengthen the bunches.

An effective control on the longitudinal bunch size is obtained by changing the voltage slope seen by every bunch at each turn by means of a higher harmonic cavity[3].

In this note we shall analyze the effect of a higher harmonic cavity on the longitudinal dynamics and estimate the requirements for bunch length control. 
We assume that the voltage of the main cavity seen by a particle is:

$$
V(\tau)=\hat{V}_{g} \cos \left(-\omega \tau+\varphi_{s o}\right)
$$

where $\hat{V}_{g}$ is the RF peak voltage, and $\tau$ is the time distance from the synchronous particle (taken as positive ahead of the bunch: $\tau=-t$ ). From eq.(2), when $\tau=0$, we have the synchronous phase $\varphi_{\text {so }}$ determined by the conditions:

$$
\cos \left(\varphi_{s o}\right)=\frac{\left(U_{o} / e\right)}{\hat{V}_{g}} \quad \sin \left(\varphi_{s o}\right)>0
$$

where $U_{o}$ is the energy loss per turn. The voltage induced by the beam in the fundamental mode is considered to be perfectly compensated by a feedback system.

\section{Active control of the bunch length}

An active external power supply controls the voltage of the higher harmonic cavity. As in the main cavity, the voltage induced by the beam is supposed to be compensated so that for the higher harmonic cavity we have:

$$
V_{n}(\tau)=k \hat{V}_{g} \cos \left(-n \omega \tau+n \varphi_{o}\right)
$$

( $n=3$-> third harmonic cavity).

Under this condition there are two free parameters that allow bunch length control: $k$ (the ratio between the peak voltages of the two cavities) and the initial phase $n \varphi_{o}$.

Since the bunch length is determined by the slope of the total voltage, the best efficiency is reached when $V_{n}(0)$ has the maximum slope, that is:

$$
n \varphi_{o}=(2 m+1) \frac{\pi}{2} \quad->\quad V_{n}(0)=0
$$

$(m=1,2,3 \ldots)$.

It is easy to see that even values of $m$, which increase the negative slope of the voltage, shorten the bunch, while odd values act in the opposite way. 
We shall evidence this behaviour by putting a double sign \pm in front of $k$, the upper one referring to the shortening regime. The total voltage is then:

$$
V(\tau)=\hat{V}_{g}\left[\cos \left(-\omega \tau+\varphi_{s o}\right) \pm k \sin (n \omega \tau)\right]
$$

In Fig. 1 we show the voltages assuming $k=.2$ and $n=3$ in the lengthening regime. Under condition (5) the higher harmonic cavity does not accelerate the bunch and the length can be controlled by changing $k$.

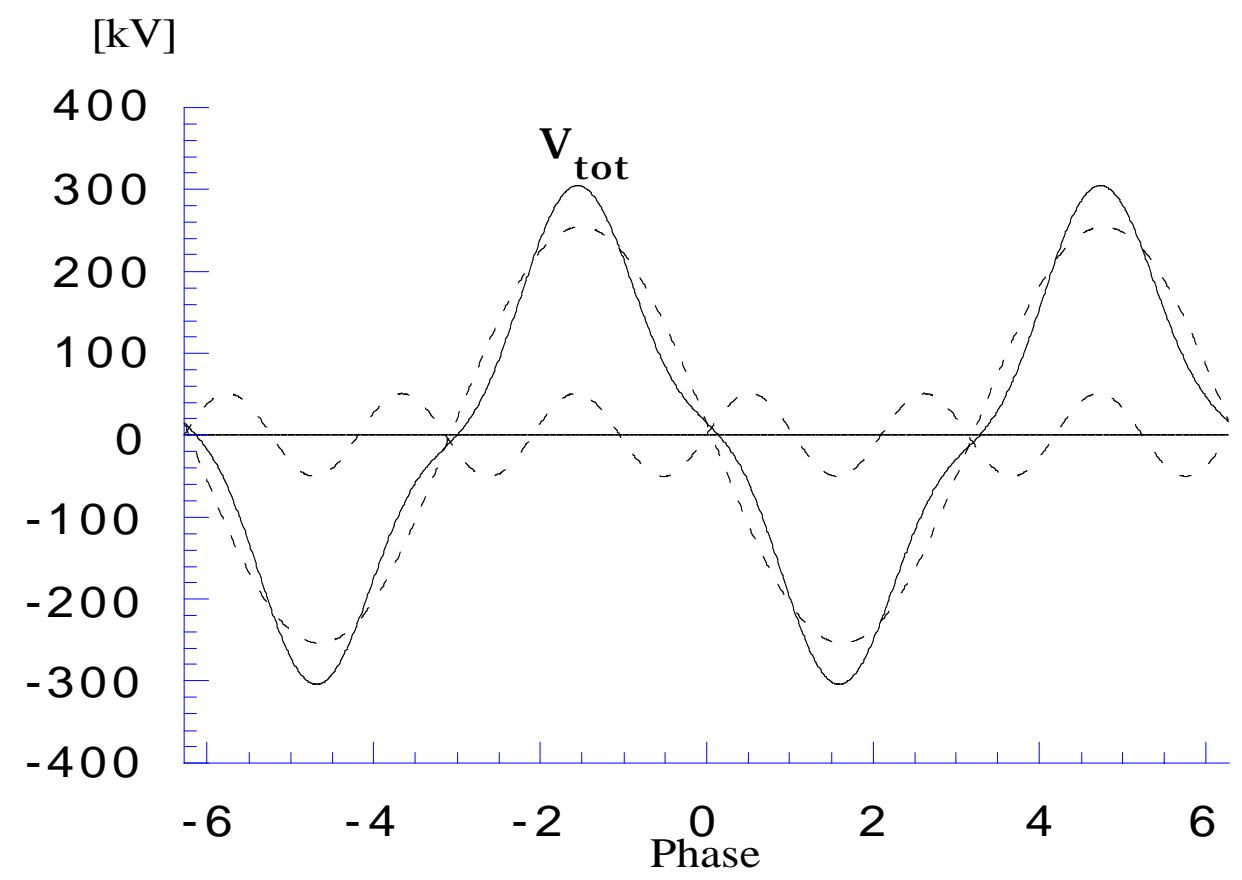

Fig. 1: Voltages with a third harmonic cavity in the lengthening regime with $k=.2$. that:

In order to provide phase stability, the synchronous phase must be such

$$
\left.\frac{d V(\tau)}{d \tau}\right|_{\tau=0}>0
$$

which implies:

$$
\mathrm{m} \frac{k n}{\sin \varphi_{s o}}<1
$$

This condition could not be satisfied in the lengthening regime when:

$$
k>\frac{\sin \varphi_{s o}}{n}
$$


However it is easy to see from the plot of the potential well shown in Fig. 2 that under this condition there exist two "mini-buckets" close to the synchronous phase, and the bunch shape is strongly deformed with two 'horns' and strong lengthening (Fig. 3).

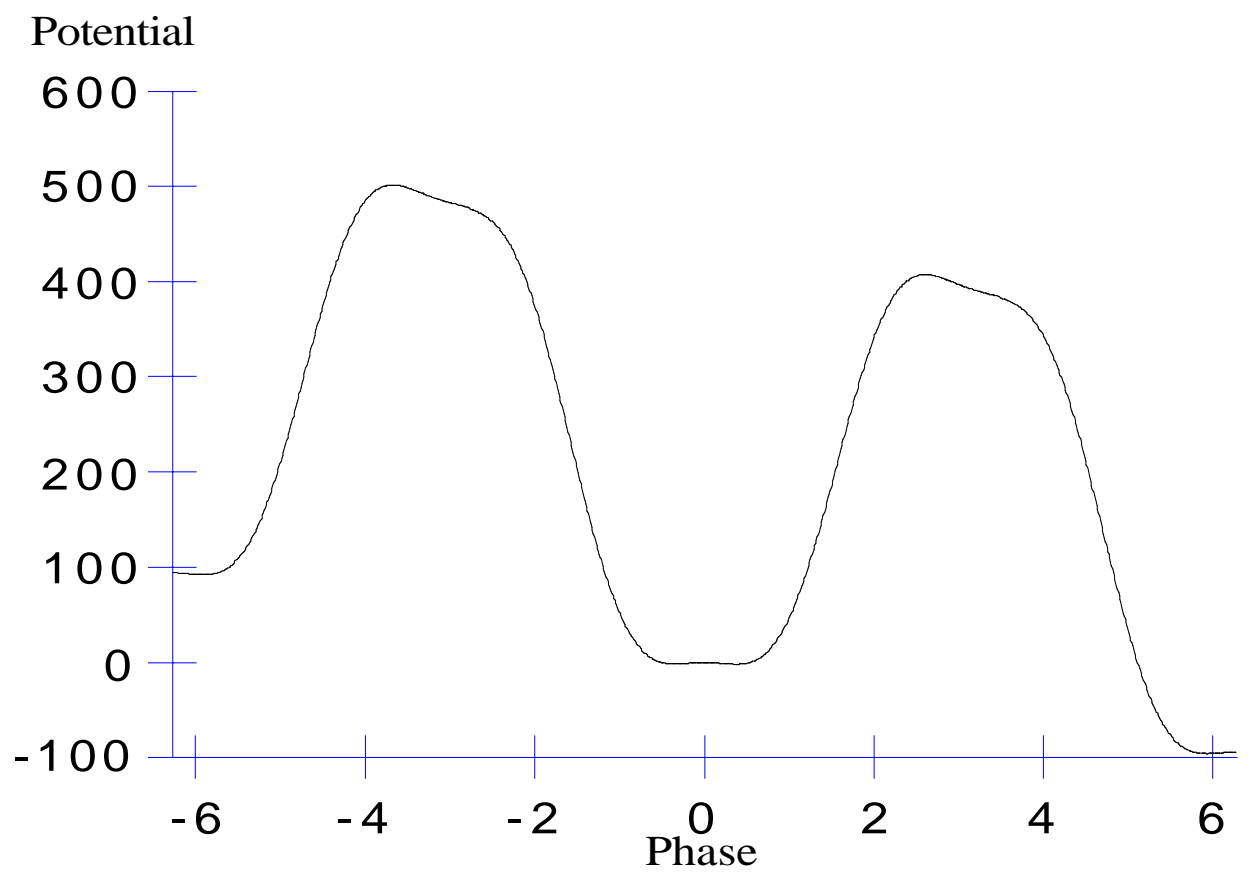

Fig. 2: Potential well for $n=3$ and $k=.4$ in arbitrary units.

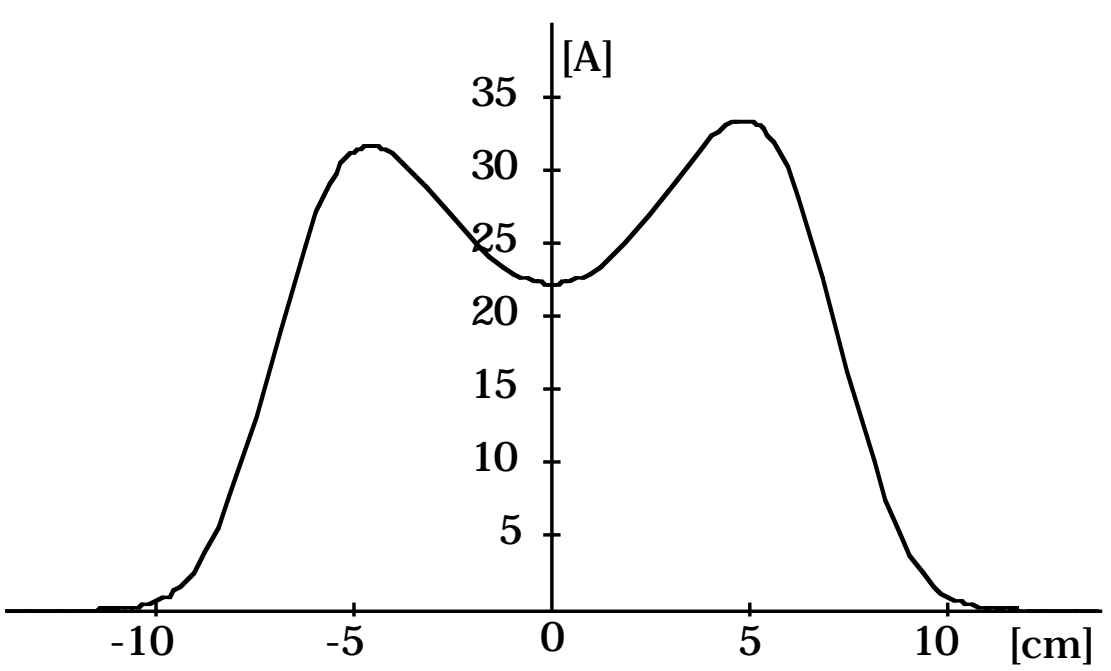

Fig. 3: Longitudinal bunch shape with $n=3$ and $k=.4$. 
From eq.(6), we obtain the potential:

$$
\begin{aligned}
\varphi(\tau)= & \frac{\alpha_{c}}{(E / e) T_{o}}\left[\frac{\hat{V}_{g}}{\omega} \sin \left(\varphi_{s o}\right) \pm \frac{k \hat{V}_{g}}{n \omega}-\frac{\hat{V}_{g}}{\omega} \sin \left(\varphi_{s o}-\omega \tau\right)+\right. \\
& \left.\mathrm{m} \frac{k \hat{V}_{g}}{n \omega} \cos (n \omega \tau)-\left(U_{o} / e\right) \tau\right]
\end{aligned}
$$

with $\alpha_{c}$ the momentum compaction, $E$ the nominal energy of the particles, and $T_{o}$ the revolution period.

\section{- Linear approximation.}

In the linear approximation $(\omega \tau<<1)$ we have:

$$
\varphi(\tau)=\frac{\alpha_{c}}{(E / e) T_{o}} \hat{V}_{g} \omega \sin \left(\varphi_{s o}\right)\left[1 \pm \frac{k n}{\sin \left(\varphi_{s o}\right)}\right] \frac{\tau^{2}}{2}
$$

From Haissinski's equation[2] in absence of turbulence regime and in the linear approximation, it is possible to make a rough estimate of the power required by the cavity to lengthen the bunch. In fact we can write:

$$
I(\tau)=I_{o} \exp \left[-\frac{1}{\sigma_{o}^{2}}\left(1 \pm \frac{k n}{\sin \varphi_{s o}}\right) \frac{\tau^{2}}{2}\right]
$$

where $\sigma_{o}$ is the rms size of the bunch distribution in the time domain due to the main cavity only. From eq.(12) we get:

$$
\sigma=\frac{\sigma_{o}}{\sqrt{1 \pm \frac{k n}{\sin \varphi_{s o}}}}
$$

It is worth noting that the same relation could be obtained by considering that [4]:

$$
\frac{\sigma}{\sigma_{o}}=\sqrt{\frac{\dot{V}}{\dot{V}_{o}}}
$$


The power required by the higher harmonic cavity is:

$$
P=\frac{k^{2} \hat{V}_{g}^{2}}{2 R_{S}}
$$

with $R_{s}$ the shunt resistance of the first fundamental mode. Substituting $k$ from eq.(15) into eq.(13) we obtain:

$$
\sigma=\frac{\sigma_{O}}{\sqrt{1 \pm \frac{n \sqrt{2 R_{S} P}}{\hat{V}_{g} \sin \varphi_{S o}}}}
$$

which relates the bunch length with the power fed to the higher harmonic cavity.

Under the assumption $\omega \tau<<1$, the RF acceptance is [3]:

$$
\Delta E_{\text {tot }}=\Delta E_{\text {main }} \sqrt{1 \pm \frac{k}{n}}
$$

As it can be easily calculated, for a third harmonic cavity with $k=\frac{\sin \left(\varphi_{s o}\right)}{n}$ and $\sin \left(\varphi_{s o}\right) \approx 1$, the change in RF acceptance due to the introduction of the higher harmonic cavity does not exceed $\pm 6 \%$.

\section{- Exact equations.}

The equations of the linear approximation are no longer valid when $k$ gets close to $\frac{1}{n}$ because other terms in the expansion of (10) must be taken into account. In general, we must use the exact equation:

$$
\begin{gathered}
I(\tau)=I_{o} \exp \left\{\frac{1}{\sigma_{o}^{2} \omega^{2} \sin \varphi_{s o}} *\right. \\
\left.*\left[\sin \left(\varphi_{s o}-\omega \tau\right) \pm \frac{k}{n} \cos (n \omega \tau)+\frac{U_{O}}{\hat{V}_{g}} \omega \tau\right]\right\}
\end{gathered}
$$


where:

$$
I_{o}=\frac{Q_{b}}{\int_{-\infty}^{+\infty} \exp \left\{\frac{1}{\sigma_{o}^{2} \omega^{2} \sin \varphi_{s o}}\left[\sin \left(\varphi_{s o}-\omega \tau\right) \pm \frac{k}{n} \cos (n \omega \tau)+\frac{U_{o}}{\hat{V}_{g}} \omega \tau\right]\right\} d \tau}
$$

with $Q_{b}$ the total charge of a bunch. The rms bunch length is defined by:

$$
\sigma=\sqrt{\frac{1}{Q_{b}} \int_{-\infty}^{+\infty} I(\tau) \tau^{2} d \tau}
$$

In Fig. 4 we plot the normalized bunch length versus $\mathrm{k}$ in the case of a third harmonic cavity at different values of $\sigma_{o}$. As obtained in the linear approximation, for short bunches the ratio $\sigma / \sigma_{o}$ does not depend on $\sigma_{o}$, while for longer bunches $\sigma / \sigma_{O}$ is rather sensitive to $\sigma_{o}$ :

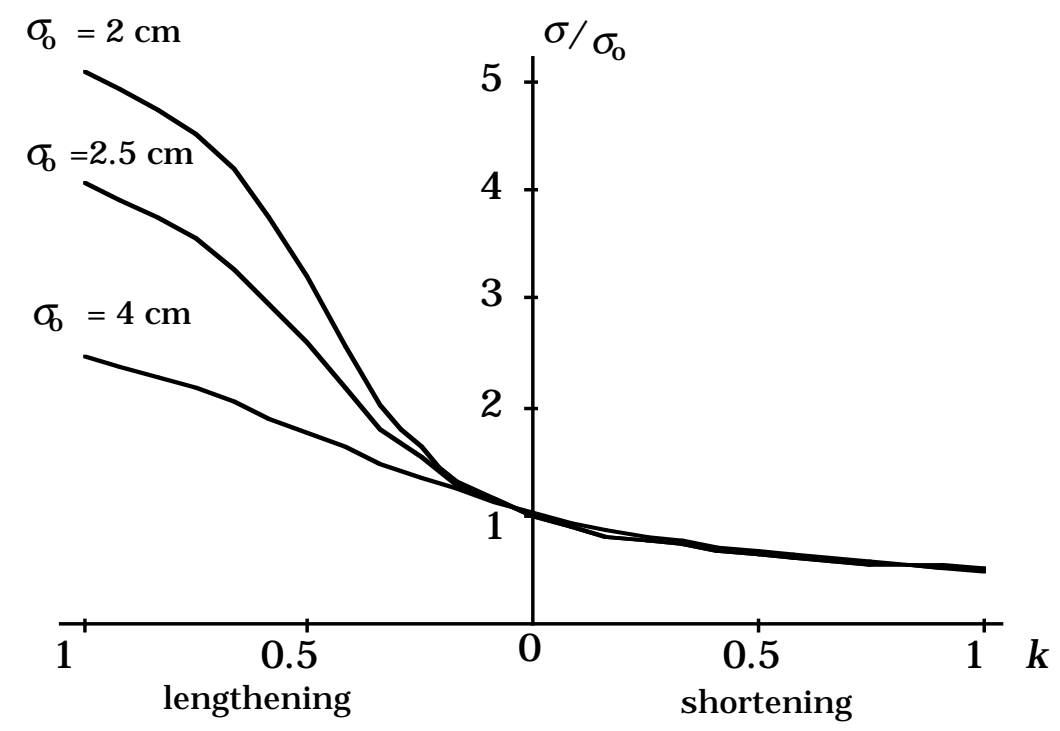

Fig. 4: Bunch length versus $k$ with a third harmonic cavity.

Finally we must mention a particular case of active bunch length control considered for LEP [5,6], where the parameters $k$ and $n \varphi_{o}$ are determined by the conditions that maximize the bunch length:

$$
\begin{aligned}
& \left.\frac{d V(\tau)}{d \tau}\right|_{\tau=0}=0 \\
& \left.\frac{d^{2} V(\tau)}{d \tau^{2}}\right|_{\tau=0}=0
\end{aligned}
$$


In Fig. 5 we show the voltages obtained under these conditions in the case of a third harmonic cavity. Anyway this situation gives only a fixed bunch length with no possibility of variation.

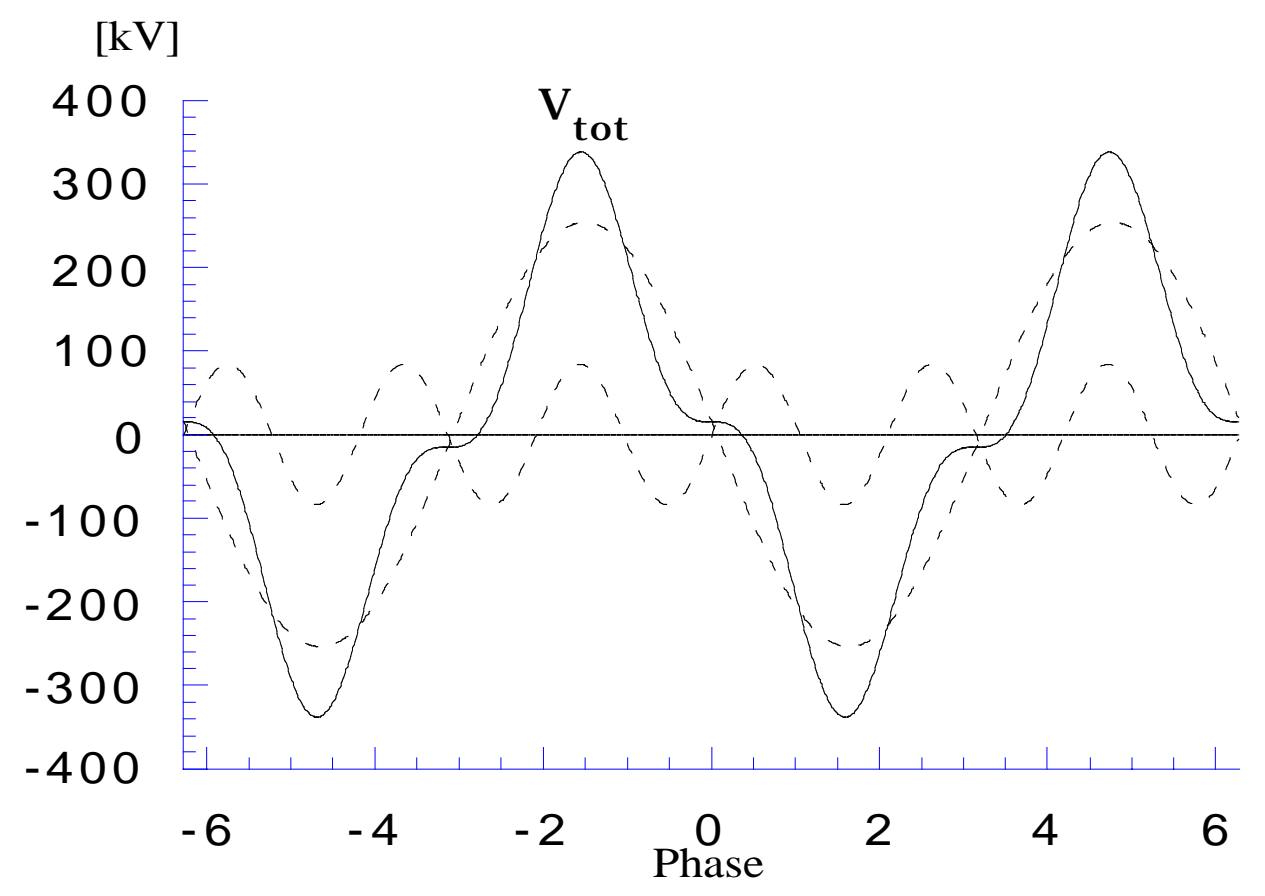

Fig. 5: Voltages in case of zero slope at the synchronous phase.

\section{Passive control of the bunch length}

In a passive higher harmonic cavity (with no external power supply), the voltage is induced by the beam itself and technical complexity and cost are greatly reduced[7].

Let us assume that $N$ bunches of charge $Q_{b}$ are stored in the machine, and let us consider only the term of frequency $n \omega$ in the Fourier expansion of the current ( $n=3->$ third harmonic cavity), i.e. the only current spectrum line that couples to the fundamental mode impedance:

$$
i_{b}(\tau)=-2 \frac{\left|Q_{b}\right| N}{T_{o}} \cos (n \omega \tau)
$$


If we define:

$$
V_{b}=\frac{\left|Q_{b}\right| N}{T_{o}} R_{S}
$$

the induced voltage is[8]:

$$
V_{b}(\tau)=-2 V_{b} \cos \left(\phi_{y}\right) \cos \left(n \omega \tau+\phi_{y}\right)
$$

where:

$$
\phi_{y}=\operatorname{arctg}\left[Q\left(\frac{n \omega}{\omega_{r}}-\frac{\omega_{r}}{n \omega}\right)\right]
$$

with $Q$ and $\omega_{r}$ respectively the quality factor and the resonant frequency of the higher harmonic cavity.

The total voltage is then:

$$
V(\tau)=\hat{V}_{g} \cos \left(-\omega \tau+\varphi_{s}\right)-2 V_{b} \cos \left(\phi_{y}\right) \cos \left(n \omega \tau+\phi_{y}\right)
$$

In this case the higher harmonic cavity accelerates the bunch and the synchronous phase changes. The new synchronous phase is determined by the condition:

$$
\cos \varphi_{S}=\frac{\left(U_{o} / e\right)+2 V_{b} \cos ^{2} \phi_{y}}{\hat{V}_{g}}
$$

with:

$$
\sin \varphi_{S}>-\frac{2 n V_{b} \cos \phi_{y} \sin \phi_{y}}{\hat{V}_{g}}
$$

Positive values of $\phi_{y}$, by increasing the negative slope of the voltage, shorten the bunch, while negative values give the opposite result.

If (28) is not satisfied, which happens only in the lengthening regime $\left(\sin \phi_{y}<0\right)$, the potential well splits into two buckets as in the active control case (see Fig. 2). 

is:

Using the same procedure of the active control, in this case the potential

$$
\begin{aligned}
& \varphi(\tau)=\frac{\alpha_{c}}{(E / e) T_{o}}\left[\frac{\hat{V}_{g}}{\omega} \sin \left(\varphi_{s}\right)+2 \frac{V_{b}}{n \omega} \cos \phi_{y} \sin \phi_{y}+\right. \\
& \left.-\frac{\hat{V}_{g}}{\omega} \sin \left(\varphi_{s}-\omega \tau\right)-2 \frac{V_{b}}{n \omega} \cos \phi_{y} \sin \left(n \omega \tau+\phi_{y}\right)-\left(U_{o} / e\right) \tau\right]
\end{aligned}
$$

In the linear approximation we can write:

$$
\begin{aligned}
& \varphi(\tau)=\frac{\alpha_{c}}{(E / e) T_{o}} \hat{V}_{g} \omega \sin \left(\varphi_{s o}\right) * \\
& *\left[\frac{\sin \left(\varphi_{s}\right)}{\sin \left(\varphi_{s o}\right)}+\frac{2 n V_{b} \cos \phi_{y} \sin \phi_{y}}{\hat{V}_{g} \sin \left(\varphi_{s o}\right)}\right] \frac{\tau^{2}}{2}
\end{aligned}
$$

and the current is:

$$
I(\tau)=I_{o} \exp \left\{-\frac{1}{\sigma_{o}^{2}}\left[\frac{\sin \left(\varphi_{s}\right)}{\sin \left(\varphi_{s o}\right)}+\frac{2 n V_{b} \cos \phi_{y} \sin \phi_{y}}{\hat{V}_{g} \sin \left(\varphi_{s o}\right)}\right] \frac{\tau^{2}}{2}\right\}
$$

so that:

$$
\sigma=\frac{\sigma_{o}}{\sqrt{\frac{\sin \left(\varphi_{s}\right)}{\sin \left(\varphi_{s o}\right)}+\frac{2 n V_{b} \cos \phi_{y} \sin \phi_{y}}{\hat{V}_{g} \sin \left(\varphi_{s o}\right)}}}
$$

The power dissipated by the beam in the higher harmonic cavity, which has to be recovered by the main cavity is:

$$
P=2 \frac{V_{b}^{2}}{R_{S}} \cos ^{2} \phi_{y}=2\left(\frac{N Q_{b}}{T_{o}}\right)^{2} R_{S} \cos ^{2} \phi_{y}
$$


therefore:

$$
\sigma=\frac{\sigma_{o}}{\sqrt{\frac{\sin \left(\varphi_{s}\right)}{\sin \left(\varphi_{s o}\right)}+\frac{n \sqrt{2 R_{S} P} \sin \phi_{y}}{\hat{V}_{g} \sin \left(\varphi_{s o}\right)}}}
$$

If the dissipated power is small, so that the relation:

$$
2 V_{b} \cos ^{2} \phi_{y}=\frac{P}{\left(\frac{N\left|Q_{b}\right|}{T_{o}}\right)}<<U_{o}
$$

is verified, then the synchronous phase remains approximately unchanged, that is:

$$
\frac{\sin \left(\varphi_{S}\right)}{\sin \left(\varphi_{s o}\right)} \approx 1
$$

To reduce the power and, at the same time, change significantly the bunch length, from (33) and (34) we must increase the shunt impedance as much as possible and detune the cavity so that $\psi_{y} \approx \pm \frac{-}{2}$. Under this condition we can write:

$$
\sin \phi_{y}= \pm 1
$$

and (34) becomes:

$$
\sigma=\frac{\sigma_{o}}{\sqrt{1 \pm \frac{n \sqrt{2 R_{S} P}}{\hat{V}_{g} \sin \left(\varphi_{s o}\right)}}}
$$

which is the same equation obtained with the active control.

Also in the passive cavity, when the induced voltage $V_{b} \cos \phi_{y}$ is high, we can not use the linear approximation but need to evaluate the exact Haissinski's equation

$$
I(\tau)=I_{o} \exp \left\{-\frac{(E / e) T_{o}}{\alpha_{c} \sigma_{o}^{2} \omega \hat{V}_{g} \sin \varphi_{s o}} \varphi(\tau)\right\}
$$


from which:

$$
\sigma=\sqrt{\frac{1}{Q_{b}} \int_{-\infty}^{+\infty} I(\tau) \tau^{2} d \tau}
$$

Even without the linear approximation, if the shunt impedance is high and the dissipated power is small, the potential can be written as $\left(\phi_{y}= \pm \frac{\pi}{2}, \varphi_{\mathrm{s}}=\varphi_{s o}\right)$ :

$$
\begin{aligned}
& \varphi(\tau)=\frac{\alpha_{c}}{(E / e) T_{o}}\left[\frac{\hat{V}_{g}}{\omega} \sin \left(\varphi_{s o}\right) \pm \frac{\left(2 V_{b} \cos \phi_{y} / \hat{V}_{g}\right)}{n \omega} \hat{V}_{g}+\right. \\
& \left.-\frac{\hat{V}_{g}}{\omega} \sin \left(\varphi_{s o}-\omega \tau\right) \mathrm{m} \frac{\left(2 V_{b} \cos \phi_{y} / \hat{V}_{g}\right)}{n \omega} \hat{V}_{g} \cos (n \omega \tau)-\left(U_{o} / e\right) \tau\right]
\end{aligned}
$$

which coincides with the potential obtained in the case of active control.

We can therefore conclude that for sufficiently small values of the dissipated power so that (35) is satisfied, and for very high values of shunt impedance so that, from (33) $\phi_{y}= \pm \frac{-}{2}$, both active and passive control of the higher harmonic cavity produce the same effect on bunch length.

However, we want to remind that in the passive mode the voltage $V_{b}$ depends on the stored current, so that the effects on the bunch length may change, and with few bunches it may be impossible to reach the desired length. Another difference arises in the case of the lengthening regime for which the tuning angle $\phi_{y}$ is negative and the Robinson instability induces dipole oscillations.

\section{Landau damping}

In order to control the bunch length we have to change the slope of the total voltage. As a consequence, the higher harmonic cavity introduces some non-linearities on the restoring force acting on the single particle, which increase the synchrotron frequency spread, thus stabilizing the coherent oscillations through the Landau damping mechanism.

Let us consider the case of very high shunt impedance and small power, so that the total voltage can be written as:

$$
V(\tau)=\hat{V}_{g}\left[\cos \left(-\omega \tau+\varphi_{s o}\right) \pm k \sin (n \omega \tau)\right]
$$


where:

$$
k=\frac{2 V_{b} \cos \phi_{y}}{\hat{V}_{g}}
$$

in the case of passive control.

The equation of motion for a single particle is:

$$
\ddot{\tau}=-\frac{\alpha_{c}}{E T_{o}}\left\{e \hat{V}_{g}\left[\cos \left(\varphi_{s o}-\omega \tau\right) \pm k \sin (n \omega \tau)\right]-U_{o}\right\}
$$

By using a perturbative method[9], it is possible to obtain the solution of (44) and the frequency of the oscillation (synchrotron frequency) in terms of power of the maximum amplitude of the first fundamental oscillation harmonic. If we call this amplitude $\hat{\tau}$ and limit ourselves to the first five terms, the solution of (44) is:

$$
\begin{aligned}
& \tau(t)=\hat{\tau} \cos (\psi)+\frac{\hat{\tau}^{2}}{4} \frac{\operatorname{cotg} \varphi_{s o}}{\left(1 \pm \frac{k n}{\sin \varphi_{s o}}\right)}\left[1-\frac{1}{3} \cos (2 \psi)\right]+ \\
& -\frac{\hat{\tau}^{3}}{192} \frac{\left(1 \pm \frac{k n^{3}}{\sin \varphi_{s o}}\right)}{\left(1 \pm \frac{k n}{\sin \varphi_{s o}}\right)} \cos (3 \psi)+\frac{\hat{\tau}^{4}}{16} \frac{\operatorname{cotg} \varphi_{s o}}{\left(1 \pm \frac{k n}{\sin \varphi_{s o}}\right)}\left[-\frac{1}{4}+\frac{1}{9} \cos (2 \psi)+\right. \\
& \left.+\frac{1}{180} \cos (4 \psi)\right]+\frac{\hat{\tau}^{5}}{3072} \frac{\left(1 \pm \frac{k n^{5}}{\sin \varphi_{s o}}\right)}{\left(1 \pm \frac{k n}{\sin \varphi_{s o}}\right)}\left[\cos (3 \psi)+\frac{1}{15} \cos (5 \psi)\right]+O\left(\hat{\tau}^{5}\right)
\end{aligned}
$$

where:

$$
\psi=\omega_{s} t+\theta_{O}
$$


with $\omega_{s}$ the synchrotron frequency, $\theta_{o}$ a starting condition, and:

$$
\begin{aligned}
& \omega_{s}(\hat{\tau})=\omega_{s o} \sqrt{1 \pm \frac{k n}{\sin \varphi_{s o}}}\left\{1-\frac{(\omega \hat{\tau})^{2}}{16\left(1 \pm \frac{k n}{\sin \varphi_{s o}}\right)}\left[\left(1 \pm \frac{k n^{3}}{\sin \varphi_{s o}}\right)+\right.\right. \\
& \left.+\frac{5}{3} \frac{\cot \operatorname{g}^{2} \varphi_{s o}}{\left(1 \pm \frac{k n}{\sin \varphi_{s o}}\right)}\right]+\frac{(\omega \hat{\tau})^{4}}{64\left(1 \pm \frac{k n}{\sin \varphi_{s o}}\right)}\left[\frac{1}{6}\left(1 \pm \frac{k n^{5}}{\sin \varphi_{s o}}\right)+\right. \\
& \left.\left.-\frac{5}{48} \frac{\left(1 \pm \frac{k n^{3}}{\sin \varphi_{s o}}\right)^{2}}{\left(1 \pm \frac{k n}{\sin \varphi_{s o}}\right)}+\frac{7}{6} \frac{\cot \mathrm{g}^{2} \varphi_{s o}}{\left(1 \pm \frac{k n}{\sin \varphi_{s o}}\right)}\right]+0\left(\hat{\tau}^{5}\right)\right\}
\end{aligned}
$$

with

$$
\omega_{s o}=\sqrt{\frac{\alpha_{c} \omega e V_{g} \sin \varphi_{s o}}{E T_{o}}}
$$

To calculate the Landau damping effect, we have to use (47) into the dispersion integral and evaluate the corresponding longitudinal impedance at the stability boundary[10]. If we want to estimate the accuracy of our approximation and the possibility of neglecting also fourth power terms in $\omega \hat{\tau}$ in (47), we can proceed as follows.

Let us assume to have a Gaussian distribution and consider the average of the synchrotron frequency weighted over the bunch distribution, namely:

$$
<\omega_{s}>=\frac{2}{\sqrt{2 \pi} \sigma} \int_{o}^{\infty} \omega_{s}(\hat{\tau}) \exp \left[-\frac{\hat{\tau}^{2}}{2 \sigma^{2}}\right] d \hat{\tau}
$$


Using (47) in the integral, we obtain:

$$
\begin{aligned}
& <\omega_{s}>=\omega_{s o} \sqrt{1 \pm \frac{k n}{\sin \varphi_{s o}}}\left\{1-\frac{(\omega \sigma)^{2}}{16\left(1 \pm \frac{k n}{\sin \varphi_{s o}}\right)}\left[\left(1 \pm \frac{k n^{3}}{\sin \varphi_{s o}}\right)+\right.\right. \\
& \left.+\frac{5}{3} \frac{\cot ^{2} \varphi_{s o}}{\left(1 \pm \frac{k n}{\sin \varphi_{s o}}\right)}\right]+\frac{3(\omega \sigma)^{4}}{64\left(1 \pm \frac{k n}{\sin \varphi_{s o}}\right)}\left[\frac{1}{6}\left(1 \pm \frac{k n^{5}}{\sin \varphi_{s o}}\right)+\right. \\
& \left.\left.-\frac{5}{48} \frac{\left(1 \pm \frac{k n^{3}}{\sin \varphi_{s o}}\right)^{2}}{\left(1 \pm \frac{k n}{\sin \varphi_{s o}}\right)}+\frac{7}{6} \frac{\operatorname{cotg}^{2} \varphi_{s o}}{\left(1 \pm \frac{k n}{\sin \varphi_{s o}}\right)}\right]+0\left(\sigma^{5}\right)\right\}
\end{aligned}
$$

Given $k, n, \sigma$, and $\varphi_{\text {so }}$, we can compare the terms of different powers in $\omega \hat{\tau}$ and see which order we can neglect. In case of a third harmonic cavity, the terms inside the square brackets are approximately of the same order of magnitude, so that the difference between the second and the fourth power of $\omega \hat{\tau}$ depends only on the bunch length. For DAФNE $(\sigma=3 \mathrm{~cm} \rightarrow \omega \sigma=.23 \mathrm{rad})$ the difference is a factor 20 , and therefore we limit ourselves to the second power of $\omega \hat{\tau}$. If we take also $\operatorname{cotg} \varphi_{\text {so }}=0$, it is possible to compare the Landau damping effect without and with the higher harmonic cavity. In the first case we write:

$$
\omega_{s}=\omega_{s o}\left[1-\frac{(\omega \hat{\tau})^{2}}{16}\right]
$$

from which we get the relation

$$
Z(p)=\frac{p(E / e) \pi \omega_{s o}^{2} \sigma^{2} \omega^{2}}{4 I_{b} \alpha_{c}}\left\{\frac{\pi e^{-y} J_{m}^{2}\left[p \omega_{o} \sigma \sqrt{2 y}\right]+j \mathfrak{I}_{p v}}{\mathfrak{I}_{p v}^{2}+\left(\pi e^{-y} J_{m}^{2}\left[p \omega_{o} \sigma \sqrt{2 y}\right]\right)^{2}}\right\}
$$


where $Z(p)$ is the maximum longitudinal shunt impedance at the stability boundary, $p$ is an integer number, $I_{b}$ the average beam current, $\omega_{o}$ the revolution angular frequency, $J_{m}$ the Bessel function of the first kind of the $\mathrm{m}^{\text {th }}$ order, $\mathfrak{I}_{p v}$ the principal value of the integral:

$$
\mathfrak{I}_{p v}=P . V . \int_{o}^{\infty} \frac{e^{-x} J_{m}^{2}\left[p \omega_{o} \sigma \sqrt{2 x}\right]}{x-y} d x
$$

and $y$ a function which depends on the coherent synchrotron frequency.

To obtain the stability region, we plot the real and the imaginary part of $Z(p)$ with the real part of y varying from 0 to $\infty$ and the imaginary part equal to $\mathrm{O}^{+}$. The stability region in this case is shown in Fig. 6 for the dipole mode of oscillation and for $p=500$.

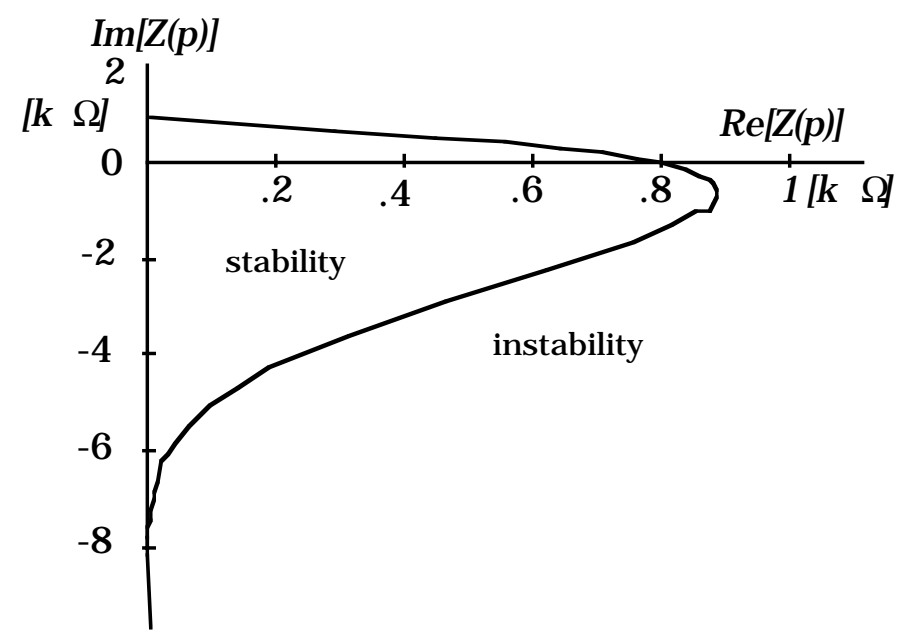

Fig. 6: Stability region for the dipole mode of oscillation with $p=500$.

In presence of the higher harmonic cavity, we obtain:

$$
\begin{aligned}
& Z(p)=\frac{p(E / e) \pi \omega_{s o}^{2} \sigma^{2} \omega^{2}}{4 I_{b} \alpha_{c}}\left(1 \pm \frac{k n^{3}}{\sin \varphi_{s o}}\right) * \\
& *\left\{\begin{array}{l} 
\pm \pi e^{-y} J_{m}^{2}\left[p \omega_{o} \sigma \sqrt{2 y}\right]+j \mathfrak{I}_{p v} \\
\mathfrak{I}_{p v}^{2}+\left(\pi e^{-y} J_{m}^{2}\left[p \omega_{o} \sigma \sqrt{2 y}\right]\right)^{2}
\end{array}\right\}
\end{aligned}
$$

where the sign + or - in the double bracket depends on whether the term $\left(1 \pm \frac{k n^{3}}{\sin \varphi_{s o}}\right)$ is positive or negative respectively. 
When:

$$
\left(1 \pm \frac{k n^{3}}{\sin \varphi_{\text {so }}}\right)>0
$$

from (54) we get the same stability region obtained with (52) but amplified by the factor $\left(1 \pm \frac{k n^{3}}{\sin \varphi_{s o}}\right)$ as shown in Fig. 7. On the other hand, if:

$$
\left(1 \pm \frac{k n^{3}}{\sin \varphi_{\text {so }}}\right)<0
$$

the stability region is upside down and amplified by the same factor, as we see in Fig. 8.

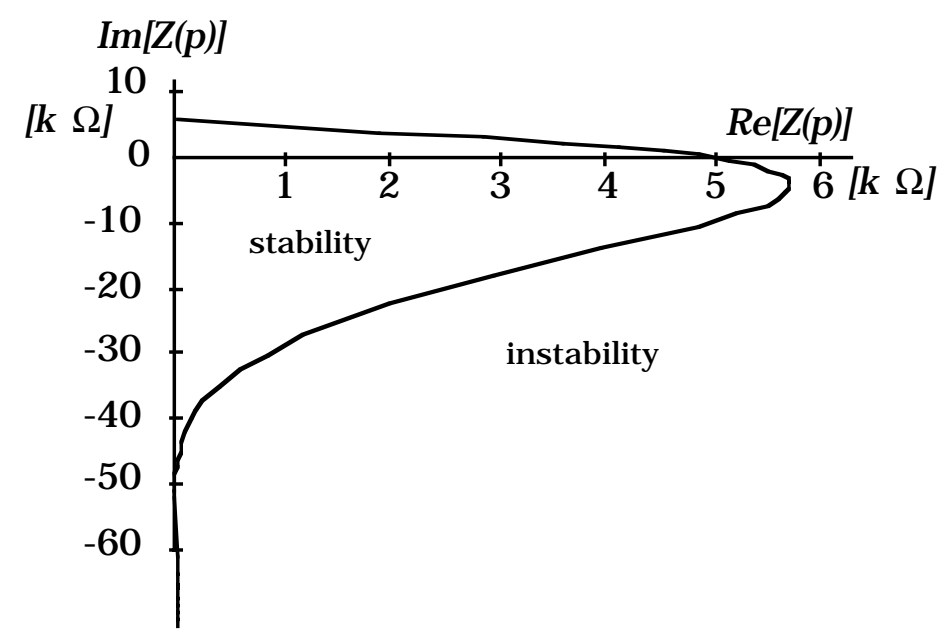

Fig. 7: Stability region with $p=500, k=.2, n=3$ in the shortening regime.

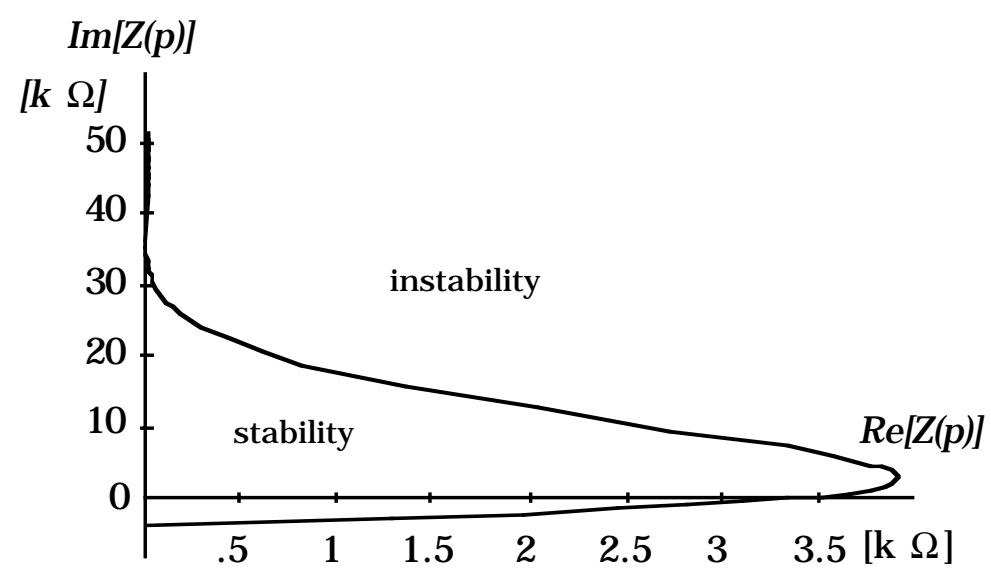

Fig. 8: Stability region with $p=500, k=.2, n=3$ in the lengthening regime. 
We can therefore conclude that in presence of a higher harmonic cavity, limiting ourselves to the second power of $\omega \hat{\tau}$ in the expression of $\omega_{S}(\tau)$, we gain a factor $\left(1 \pm \frac{k n^{3}}{\sin \varphi_{s o}}\right)$ on the maximum value of the allowed longitudinal HOM impedance.

\section{Application to DA $\Phi$ NE.}

In order to evaluate the bunch length in presence of the higher harmonic cavity we have first solved Haissinski's equation with vanishing broad band impedance, i.e. without any turbulence. At different values of the shunt impedance of the higher harmonic cavity fundamental mode, we plot in Fig. 9 the bunch length versus the power supplied by the higher harmonic cavity with the active control, or by the main cavity with the passive one.

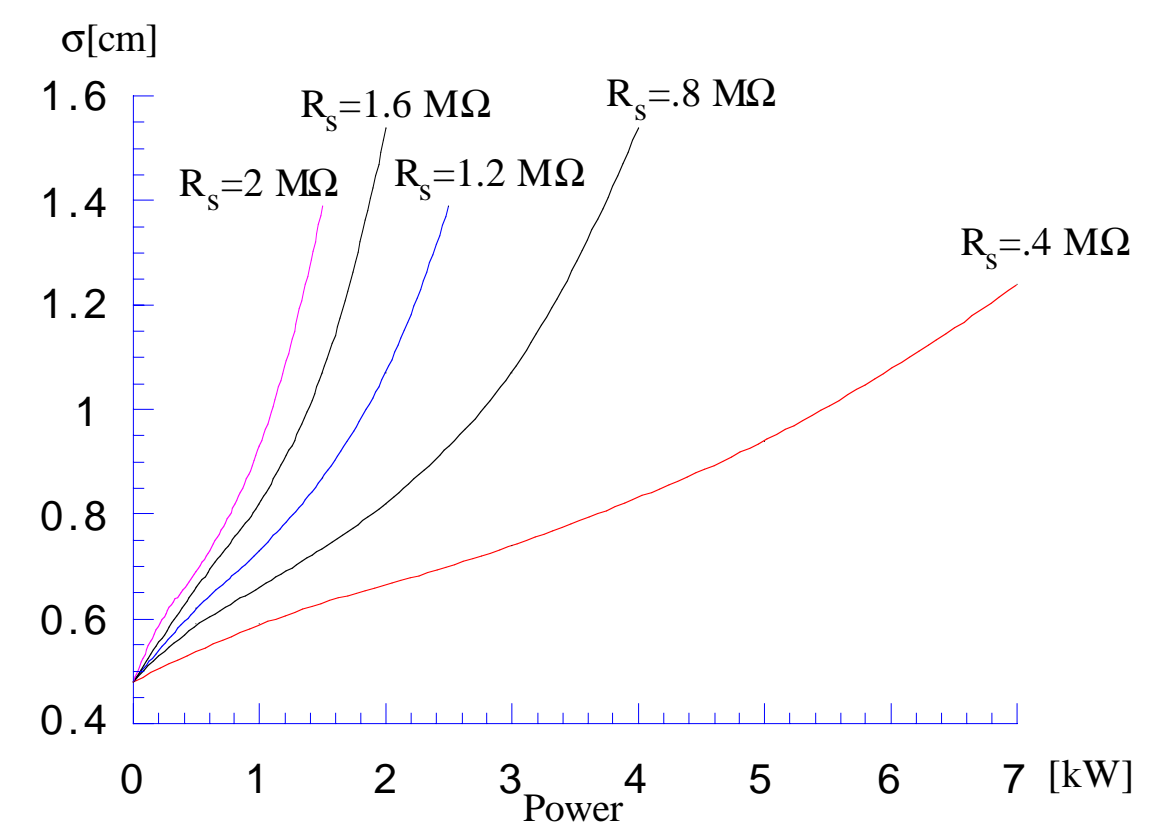

Fig. 9: Bunch length versus power without broad band impedance at different shunt resistances.

We have considered only the region where the potential well is not significantly deformed by non-linearities, so that the bunch remains practically gaussian, and the peak current required by the Boussard criterion can be written as[2]:

$$
\hat{I}=\frac{\left|Q_{b}\right|}{\sqrt{2 \pi} \sigma}
$$


Such a peak current, in the turbulent regime, changes the energy spread. But a new value of energy spread leads to a new distribution of particles in the potential well, and therefore, a new bunch length. With Haissinski's equation we can take into account the distortion of the potential well due to the broad band impedance, and calculate the new $\sigma$. If we assume that the bunch is still gaussian so that (57) is verified, we find a new peak current which, again, influences the energy spread.

The final bunch length is obtained by solving self-consistently Boussard's criterion and Haissinski's equation together with (57).

In Fig. 10 we show the final bunch length versus power with different shunt impedances and with the longitudinal broad band impedance estimated so far for the DAФNE main rings, while in Fig. 11 we have doubled the value of the inductive component of the broad band impedance.

The two plots refer to both active and passive control of the higher harmonic cavity, because the shunt impedance is very high (from 4 to $2 \mathrm{M} \Omega$ ) and the power small (less than $7 \mathrm{~kW}$ ).

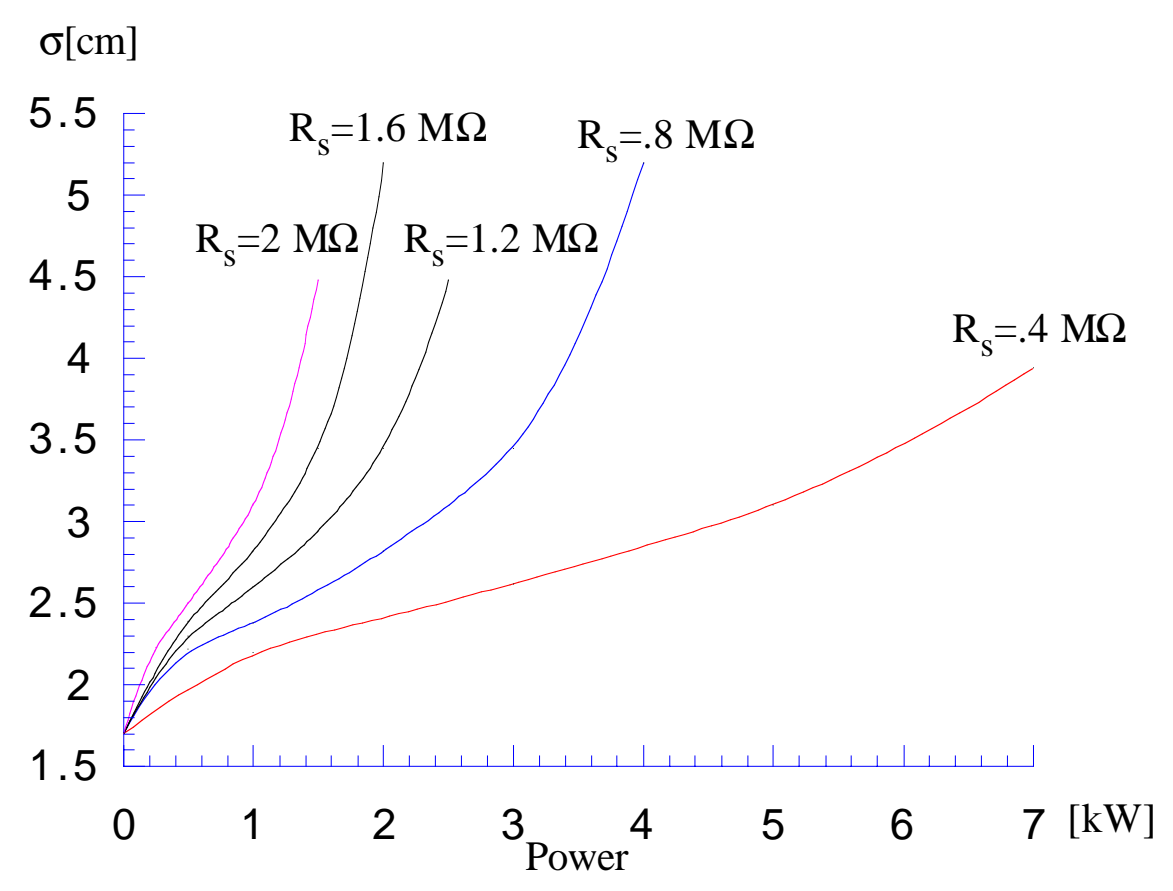

Fig. 10: Bunch length versus power at different shunt resistances with a broad band impedance of $.42 \Omega$. 


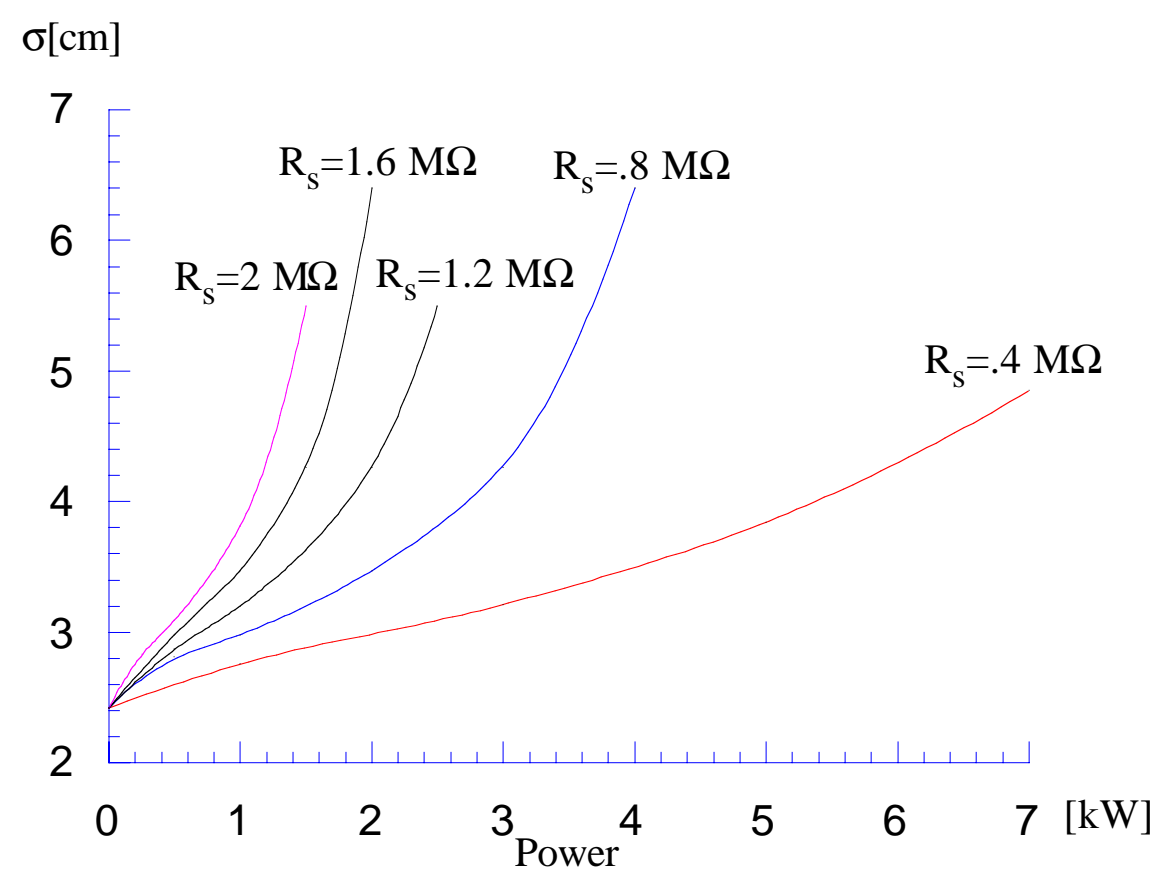

Fig. 11: Bunch length versus power at different shunt resistances with a broad band impedance of $.78 \Omega$.

\section{Conclusions.}

A higher harmonic cavity in the main ring gives an additional degree of freedom to adjust the bunch length. An effective control on the longitudinal bunch size is obtained by changing the voltage slope seen by the particles at each turn by means of such a cavity.

We have shown that active and passive control of the cavity have the same effect on the bunch length, provided the shunt impedance of the fundamental mode is very high. For DAФNE we have shown that $\approx 2 \mathrm{M} \Omega$ resistance allows to keep the bunch length under control with $1 \mathrm{~kW}$ assuming an inductive broad band impedance in the range of $0.5 \div 1 \Omega$.

Furthermore there is an enhancement of the Landau damping effect, beneficial for damping multibunch instabilities.

Another advantage comes from the fact that although the voltage slope can be changed over a wide range, the variation of $\mathrm{RF}$ acceptance does not exceed $\pm 6 \%$ for the parameters of interest. 
A passive higher harmonic cavity seems to be preferable to an active one because it greatly reduces cost and technical complexity due to the absence of an additional power source. However, we must mention that the bunch length changes with the number of bunches and with few bunches it may be impossible to reach the desired bunch length. Furthermore the Robinson instability can occur with the passive cavity in the lengthening regime, for which the tuning angle is negative.

\section{References}

[1] "Proposal for a $\Phi$-Factory", LNF-90/031(R), April 1990.

[2] M. Migliorati, et al., "Bunch Lengthening in DAథNE Main Ring", DAФNE Technical Note G-22, November 1993.

[3] L. Palumbo, "Double R.F. System for DAФNE", DAФNE Technical Note RF-2, December 1990.

[4] M. Sands, "The Physics of Electron Storage Ring. An Introduction", SLAC-121 UC-28, November 1970.

[5] A. Hofmann, S. Myers, "Calculations of RF and Beam Parameters for the Double RF System", LEP Note 158, June 1979.

[6] P. Bramham, et al., "A Higher Harmonic Cavity to Increase the Bunch Length in LEP-70", LEP/70-25, December 1977.

[7] R. A. Bosch, C. S. Hsue, "Suppression of Longitudinal Coupled-Bunch Instabilities by a Passive Higher Harmonic Cavity", Particle Accelerators, Vol.42, N. 2, 1993.

[8] M. Migliorati, Tesi di Laurea, Roma, April 1992, unpublished.

[9] N. N. Bogoliubov, Y. A. Mitropolsky, "Asymptotic Methods in the Theory of NonLinear Oscillations", Hindustan Publishing Corpn. (India), 1961.

[10] M. Migliorati, et al., "Landau Damping of Longitudinal Multibunch Instabilities in DAФNE", DAФNE Technical Note G-21, September 1993. 